\title{
EFFECT OF SOFT SEGMENT CHAIN LENGTH ON TAILORING THE PROPERTIES OF ISOCYANATE TERMINATED POLYURETHANE PREPOLYMER, A BASE
}

\author{
MATERIAL FOR POLYURETHANE BANDAGE
}

\author{
Runumi Gogoi ${ }^{1}$, M. Sarwar Alam ${ }^{2}$, Utpal Kumar Niyogi ${ }^{3}$ \\ ${ }^{1,3}$ Material Science Division, Shriram Institute for Industrial Research, 19 University Road, Delhi 110007, India \\ ${ }^{2}$ Dept of Chemistry, Jamia Hamdard University, Hamdard Nagar, Delhi - 110062, India \\ Runumi@rediffmail.com
}

\begin{abstract}
Isocyanate terminated polyurethane prepolymer, a base material for water curable polyurethane bandage was prepared from diphenylmethane diisocyanate and poly (propylene oxide) glycol. In polyurethanes, the diisocyanate act as hard segment and provides dimensional stability, whereas the polyol which forms the soft segment gives elastomeric character. Due to its segmented structure, polyurethane's with a wide range of physico-mechanical properties can be obtained by varying its monomers types and content. In this paper, the effect of different soft segment length on influencing the structure property relationship of polyurethane prepolymer was studied. The soft segment length was varied by using poly (propylene oxide) glycol having different molecular weight and also by blending two polyols having similar chemical composition but of different molecular weight. The formation of the prepolymer was investigated by Fourier transform infrared spectroscopy (FTIR). The results reveal that with increasing soft segment length, the weight average molecular weight and storage time of the prepolymer increases, whereas the Brookfield viscosity decreases due to higher content of soft segment. The curing time was found higher in prepolymer having longer soft segments due to lower isocyanate content. With increasing molecular weight of the soft segment, the phase separation between the hard and soft segment increases due to which the prepolymer produce low $T_{g}$. The tensile strength of the polyurethane film decreases whereas percent elongation increases with increase in soft segment length. The findings of the study will be beneficial for understanding the role played by soft segment length on tailoring the structure and ultimately the properties of isocyanate terminated polyurethane prepolymer.
\end{abstract}

Keywords: Polyurethane prepolymer, soft segment length, polyurethane bandage

\section{INTRODUCTION}

Polyurethanes are block copolymers composed of alternating soft and hard blocks or segment. The soft segment provides elastomeric character to the polymer while the hard segment forms dimensional stability. The soft segment with a low $\mathrm{T}_{\mathrm{g}}$ are formed from polyether or polyester, generally of mol.wt 400-5000. The rigid polar hard segments with a high $\mathrm{T}_{\mathrm{g}}$ are based on diisocyanate and low molecular weight chain extender. At room temperature, the polyol segment are above their $\mathrm{T}_{\mathrm{g}}$, so they are denoted as soft segment and the aromatic diisocyanate which are below their $\mathrm{T}_{\mathrm{g}}$ act as hard segment [1]. Because there exist a degree of thermodynamic immiscibility between the hard urethane segments and the soft polyol segments, polyurethane elastomers exhibit microphase separation, which could result in a structure that can be considered as hard segment domains dispersed in soft segment matrix. Due to the presence of two phase micro domain structure, the molecular structure of polyurethane can be tailored to meet specific property requirement by varying the hard and soft segment length and concentration [2].

The urethane linkage (-NH-COO-) in the polyurethane is a result of the reaction between the diisocyanate (-NCO) groups and polyol hydroxyl groups $(-\mathrm{OH})$. If a stoichiometric excess of diisocyanate is used, NCO terminated urethane chains are produced and the product is called isocyanate terminated prepolymer. The formation of isocyanate terminated polyurethane prepolymer and its subsequent curing reaction are represented schematically in scheme I. The curing of the prepolymer takes place by coming in contact with water to form a network of poly (urethane urea). 

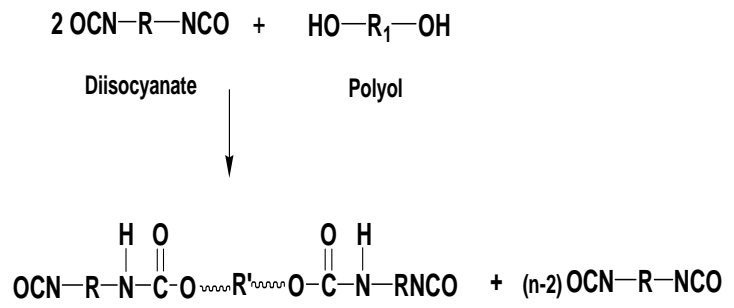

Polyurethane prepolymer
\[ \mathrm{H}_{2} \mathrm{O} \]

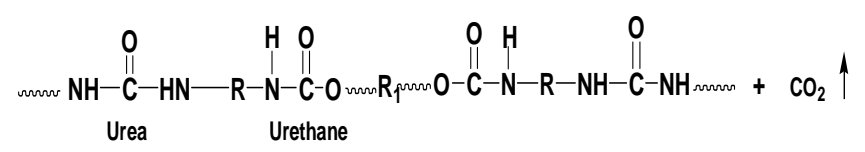

Scheme 1: Formation of isocyanate terminated polyurethane prepolymer and its curing reaction

Many studies have been done in the past to observe the structure property relationship of polyurethane. It was reported in the literature that by varying the type and amount of polyol, diisocyanate, catalyst, reaction time and temperature, polyurethanes with a wide range of physico-mechanical properties can be obtained [3]. It was also mentioned in the literature that the prepolymer formation depends upon a number of chemical factors. Among these, the degree of branching $(\mathrm{Mc})$ of polyol system and the equivalent weight of the polyether or polyester greatly influence the formation of the prepolymer [4].

The equivalent weight of polyol employed has a definite effect on the viscosity buildup during the prepolymer formation. The concentration of lower molecular weight polyol has more influence on the viscosity than the concentration of higher molecular weight polyol [3]. Also the number and size of diol molecules which condense with diisocyanate, determines the no of urethane linkages within the isocyanate terminated prepolymer [3]. And the urethane group content determines the degree of hardness and flexibility of the polyurethane [5].
In the field of coating technology, it was observed that the chemical composition and molecular weight distribution (MWD) of the incorporated soft block influence the macroscopic properties of the resulting coatings [6].

The isocyanate terminated polyurethane prepolymers synthesized in the present study will be applicable for preparing water curable polyurethane bandage and for that application, the proper choice of polyol molecular weight is necessary to have proper mechanical strength, curing time and storage stability. Thus, in the present study, a study was done to see the effect of different soft segment chain length on the physico-chemical, mechanical and thermal properties of polyurethane prepolymer. The soft segment chain length was varied by using poly (propylene oxide) glycol polyol having different molecular weight and also by blending two polyols of different molecular weight with the same chemical composition. The isocyanate terminated polyurethane prepolymer was synthesized by thermal polymerization technique using diphenyl methane diisocyanate (MDI) and poly (propylene oxide) glycol (PPG) at fixed isocyanate / hydroxyl molar ratio. Their properties viz. physico-chemical, mechanical, thermal and structures were investigated by different ASTM and IS methods, thermal analysis, tensile test and FTIR technique.

\section{EXPERIMENTAL MATERIALS}

Diphenyl methane diisocyanate (MDI) was obtained from Sigma-Aldrich, USA. A white solid flakes (melt.pt. 40oC), it was used as such without further purification. Poly (propylene oxide) glycol (PPG) was obtained from Sigma-Aldrich, USA. The polyol was vacuum dried for 2 hours at $80 \mathrm{oC}$ and kept overnight in molecular sieves prior to use to ensure that the material is free from moisture (less than 0.05\%). The properties of MDI and PPG used for the synthesis of polyurethane prepolymer are given in Table I. Catalysts 2,2' dimorpholino diethyl ether (DMDEE) from Degussa, Germany was procured and used as such without any purification.

Table 1: Some properties of MDI and PPG used for the prepolymer synthesis

\begin{tabular}{|l|c|c|c|c|c|}
\hline Property & MDI & PPG400 & PPG1000 & PPG2000 & PPG 2700 \\
\hline Average functionalitya & 2.0 & 2.0 & 2.0 & 2.0 & 1.8 \\
\hline OH number (mg KOH/g)a & 0.0 & 281 & 111 & 56 & 37 \\
\hline NCO content (wt\%)b & 33.8 & 0.0 & 0.0 & 0.0 & 0.0 \\
\hline Molecular weight (g/mol)a & 250 & 400 & 1000 & 2000 & 2700 \\
\hline Viscosity 25oC, (cSt)a & 50 & 99 & 150 & 300 & 627 \\
\hline
\end{tabular}

a Information given by supplier

b Estimated by ASTM D2572 - 97(2010) 


\subsection{Synthesis of Polyurethane (PU) Prepolymer}

The synthesis was carried out in a 5-necked glass reactor equipped with teflon stirrer, thermometer pocket, condenser and nitrogen gas inlet system. The MDI was melted in a beaker and poured into the reactor under nitrogen atmosphere. Inert atmosphere was maintained throughout the reaction so as to avoid the ingress of atmospheric moisture. The required amount of catalyst $(0.25 \%$ of the whole reaction mixture) was then added followed by addition of PPG. The PPG was added part wise to the reactor. After that, the system was maintained at $60 \mathrm{oC}$ for 2 hours. The final prepolymer obtained was in the form of a yellow colored viscous liquid.

The isocyanate terminated polyurethane prepolymers were synthesized using different molecular weight of polyol (PPG 400, PPG 1000, PPG 2000, PPG 2700) and MDI at NCO / OH molar ratio of 3:1. Mixed polyols i.e. PPG 1000 \& PPG 400 in 50 / 50 ratio and PPG 2000 \& PPG 400 in 50 / 50 ratio was also used for synthesizing the prepolymer.

\subsection{Preparation of Polyurethane Film}

Polyurethane films were prepared by casting evaporation technique. Approximately $10 \%$ solution of polyurethane prepolymer in tetrahydrofuran was prepared and poured into a Teflon mould. It was then kept at room temperature, till all the solvent got evaporated and a transparent film was formed. The films obtained were of about $0.4 \mathrm{~mm}$ thickness.

\subsection{Characterization}

The following properties were characterized in the synthesized prepolymers:

The Fourier Transform Infrared Spectroscopy (FTIR) spectra of the prepolymers was taken on an FTIR, Perkin Elmer Model 521 in the range of wavenumber from $400 \mathrm{~cm}^{-1}$ to $4000 \mathrm{~cm}^{-1}$ by coating $1 \mathrm{~mm}$ thick layer of the prepolymer on a $\mathrm{KBr}$ pellet.

The isocyanate content in the prepolymer was determined as per ASTM D 2572-91. The isocyanate present in the prepolymer was reacted with an excess of di-n-butyl amine and then titrated with standard $0.1 \mathrm{~N} \mathrm{HCl}$ solution. Since a known amount of amine was added, the amine consumed by the prepolymer is due to the presence of free isocyanate which was determined. The data given are the average of three measurements.

Dynamic viscosity of the prepolymer was determined by Brookfield viscometer DV Pro II (Brookfield Engineering Laboratories Inc, USA). The measurements were performed at $35 \pm 1^{\circ} \mathrm{C}$ at $12 \mathrm{rpm}$ using spindle No.4.

The weight average molecular weight $\left(\mathrm{M}_{\mathrm{w}}\right)$ of the prepolymer was determined by gel permeation chromatography using
2414 RI Detector (Make -Waters) and polystyrene as a standard. The samples were prepared in duplicate by dissolving $0.5 \%$ and $0.1 \%$ by wt of prepolymer in tetrahydrofuran (THF). THF was also used as a carrier solvent at the rate of $0.5 \mathrm{ml} / \mathrm{min}$.

Storage time was determined as per the method mentioned in US Patent 5,66,5056. For determining the storage stability about $50 \mathrm{ml}$ of the PU prepolymer is poured in a polypropylene bottle and hermetically sealed under nitrogen atmosphere. The bottle was kept in an oven controlled at $130^{\circ} \mathrm{C}$ and the time till the resin was flow able was recorded. An average of three measurements was taken as the result.

The set to touch time was determined according to ASTM

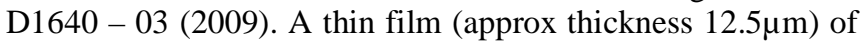
PU prepolymer was coated on a glass plate. From time to time, the tip of a clean finger was lightly touched on the test film and immediately placed the fingertip on a piece of clean, clear glass plate. Observed, if any of the prepolymer was transferred to the glass plate If no prepolymer was transferred, the time was noted as the set to touch time of the polyurethane prepolymer. The test was carried out at $30-32^{\circ} \mathrm{C}$ and relative humidity of $40-45 \%$. An average of three measurements was taken as the result.

Tack-free time was determined according to IS 101 (Part 3/Sec1) -1986. A thin film of PU prepolymer was coated on a glass plate. The plate was then placed in one pan of suitable balance and counterpoised with weights. Placed a further weight of $2.25 \mathrm{~kg}$ and press on the dried film surface of the plate with thumb till the two pans are balanced Hold for one minute and then slowly release. If no sign of stickiness appears on the thumb, the time was noted and reported as tack-free time of the prepolymer. Temperature was maintained at $30-32^{\circ} \mathrm{C}$ and relative humidity of $40-45 \%$. The data given was the average of three measurements.

The tensile strength and percent elongation of the polyurethane films were measured in a universal testing machine (Tinius Olsen, H5KL). The dimension of the test specimen was $10 \mathrm{~cm} \times 2 \mathrm{~cm} \times 0.04 \mathrm{~cm}$ and the measurement was performed under load cell of $250 \mathrm{~N}$ with a crosshead speed of $5 \mathrm{~mm} / \mathrm{min}$ at room temperature. The data taken are the average of three measurements.

Differential Scanning Calorimetry (DSC) was carried out to characterize the thermal properties of the prepolymer. The measurements were carried out under nitrogen atmosphere on a DSC - 2910 ( TA instrument). The heating rate was of $10^{\circ} \mathrm{C}$ / $\mathrm{min}$ in the temperature range of $-100^{\circ} \mathrm{C}$ to $100^{\circ} \mathrm{C}$. 


\section{RESULTS AND DISCUSSION}

\subsection{Influence of Soft Segment Length on Structure of}

\section{the Prepolymer}

Fig- 1 shows the IR spectra's of the synthesized prepolymers using different polyol molecular weight. All the synthesized prepolymer shows the characteristics absorption bands of polyurethanes except in the peak associated to $\mathrm{NCO}$ stretching which shows higher absorption area in case of lower molecular weight polyol. This is due to the presence of higher percent isocyanate content in case of lower molecular weight polyol. It was observed that all the prepolymers possess the following characteristic absorption bands, urethane NH stretching at $3300 \mathrm{~cm}^{-1}$, bending at $1516 \mathrm{~cm}^{-1}$, methylene or alkyl group at $2974 \mathrm{~cm}^{-1}$, carbonyl group at $1700 \mathrm{~cm}^{-1}$ and C-O$\mathrm{C}$ stretching at $1100 \mathrm{~cm}^{-1}$. The strong characteristic peak at $2272-2275 \mathrm{~cm}^{-1}$ is associated with NCO stretching. Presence of NCO stretching band and all the above-mentioned bands indicates formation of isocyanate terminated polyurethane prepolymer.

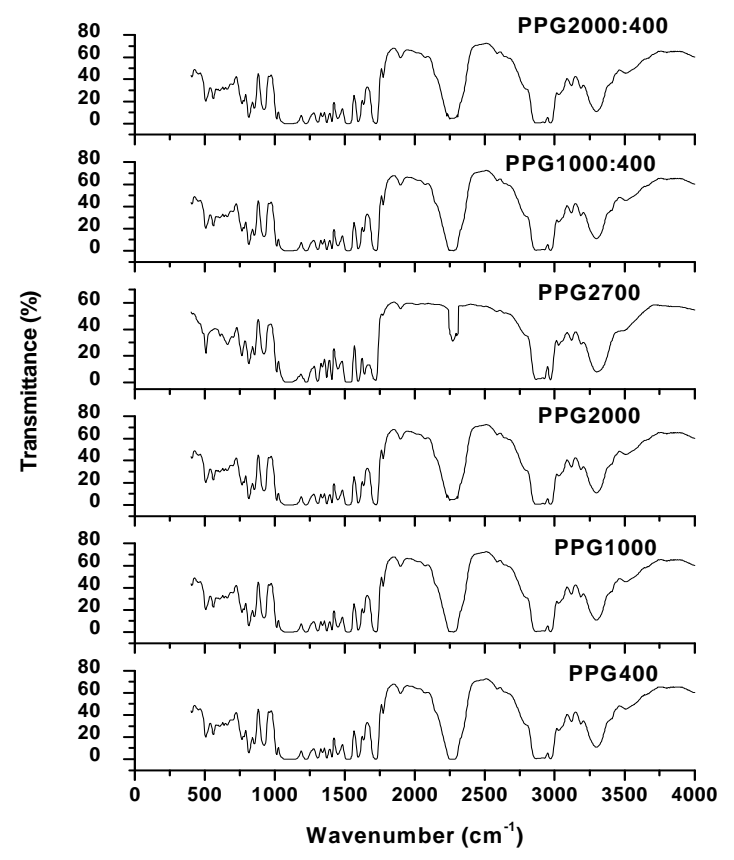

Fig-1: IR spectra of the prepolymers synthesized using different molecular weight of polyol

\subsection{Influence of Soft Segment Length on the Physico-}

\section{Chemical Properties}

The influence of different soft segment length on the physicochemical properties of isocyanate terminated polyurethane prepolymer was shown from Fig 2 to 6 . It was observed that as the molecular weight of the polyol increases, the isocyanate content of the prepolymer decreases. Mixing the polyol in 50 / 50 ratios gives isocyanate content in between their respective counterparts.

The viscosity of the prepolymer decreases with increasing the molecular weight of the polyol This is because of the fact that viscosity of polyurethane prepolymer depends on the urethane group content of the prepolymer. As the number of urethane groups increases, the $\mathrm{NH}$ group and carbonyl group of urethane linkage form strong physical crosslinking, thereby increasing the stiffness of the network causing increase in viscosity. The higher molecular weight polyol generates less urethane groups thus having less viscosity in comparison to lower molecular weight polyol which produces more urethane groups [6].

The molecular weight of the prepolymer was found to be higher in case of high molecular weight polyol due to its longer chain length.

On observing the storage stability of the prepolymer, it was found that the storage stability of the prepolymer was less in case of prepolymer synthesized by using lower molecular weight polyol. This is due to the generation of more urethane groups in case of lower molecular weight polyol which increases the physical crosslinking thereby decreasing the flowability of the prepolymer and ultimately the storage time. By mixing two polyols having different molecular weight in 50 / 50 ratio, the storage time was observed to be in between their respective counterparts.

The set to touch time and tack free time of the prepolymer was found to increase with increasing molecular weight of the polyol. This is due to the fact that lower molecular weight polyol generates more NCO terminated short chain segments, thus creating more reactive sites for reaction with water which in turn cause a decrease in set to touch time and tack free time of the prepolymer. In case of higher molecular weight polyol, less isocyanates end groups are produced for reaction with water, thus having longer curing time.

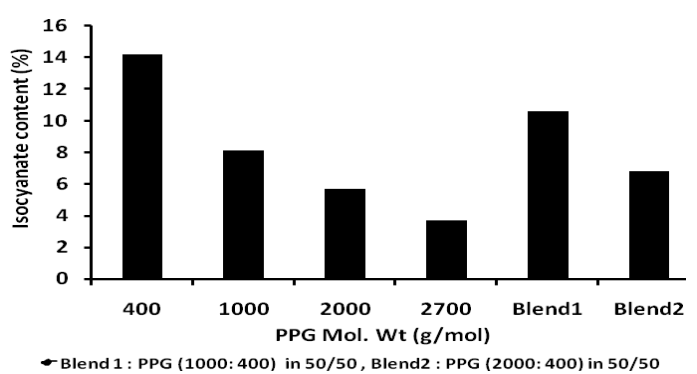

Fig-2: Effect of polyol molecular weight on isocyanate content of the prepolymer 


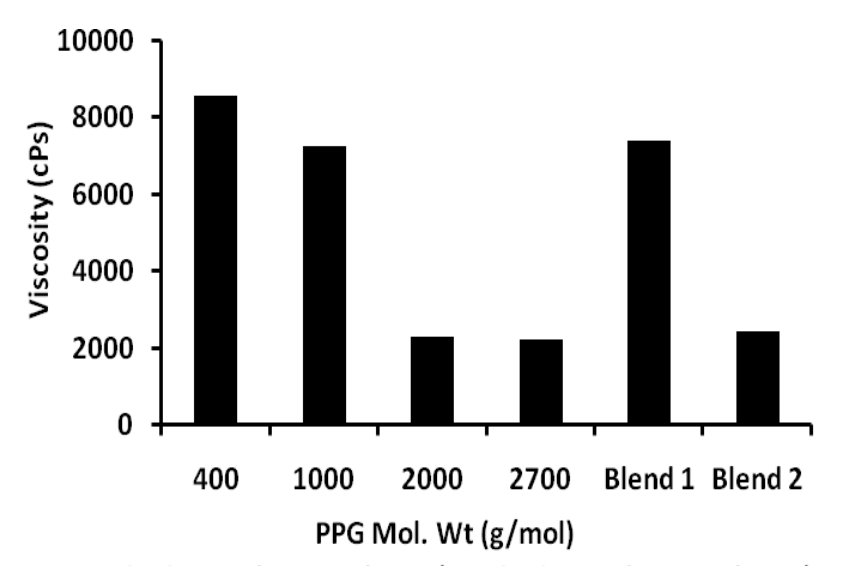

$\leftarrow$ Blend 1: PPG $(1000: 400)$ in 50/50, Blend2 : PPG $(2000: 400)$ in 50/50

Fig-3: Effect of polyol molecular weight on viscosity of the prepolymer

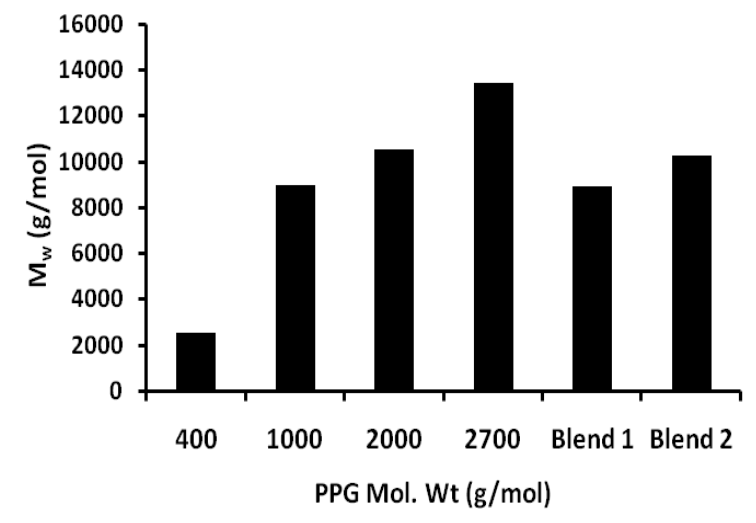

- Blend 1: PPG (1000:400) in 50/50, Blend2 : PPG (2000:400) in 50/50

Fig-4: Effect of polyol molecular weight on weight average molecular weight of the prepolymer

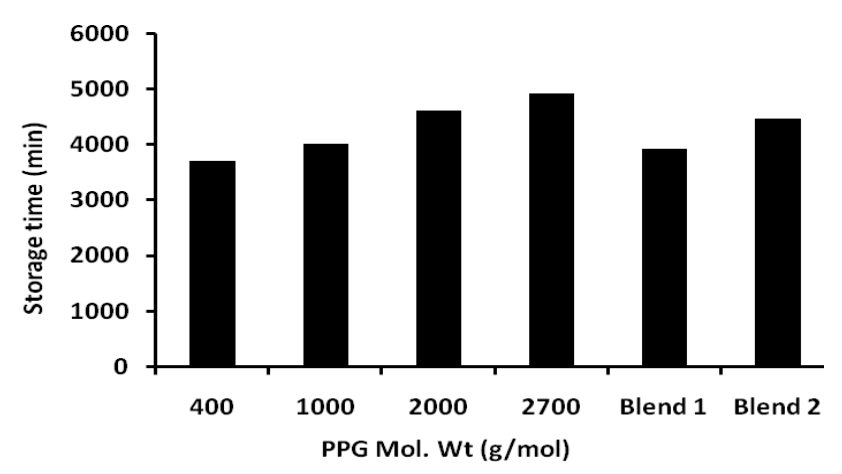

-Blend 1: PPG (1000: 400) in 50/50, Blend2 : PPG (2000:400) in 50/50

Fig-5: Effect of polyol molecular weight on storage time of the prepolymer

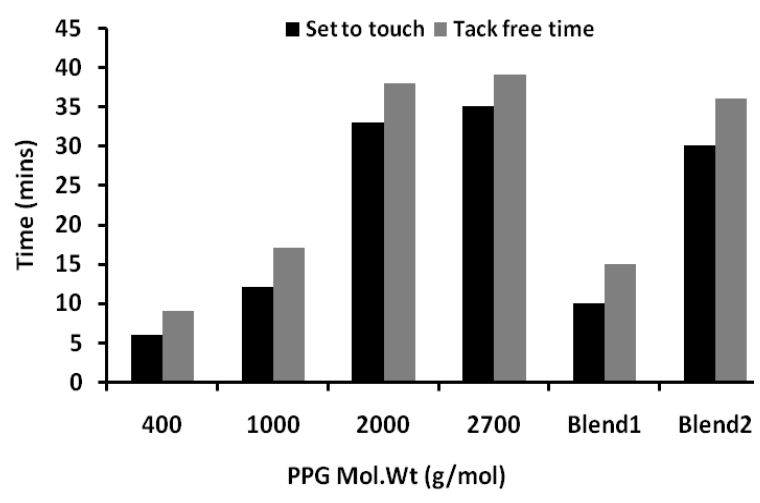

- Blend 1: PPG (1000: 400) in 50/50,Blend2 : PPG (2000:400) in 50/50

Fig-6: Effect of polyol molecular weight on curing time of the prepolymer

\subsection{Influence of Soft Segment Length on the}

\section{Mechanical Properties}

Results in Fig- 7, indicates that as the molecular weight of the polyol increases, linear decrease in tensile strength and increase in percent elongation was observed as the polymer becomes increasingly soft with increase in chain length of the soft segment. But in case of PPG 400, both the tensile strength and percent elongation was observed to be low due to the brittle nature of the polymer [7]. In case of mixed polyol i.e. PPG1000 and PPG 400 in 50 / 50 ratio, the tensile strength was found to be highest among all the polyurethane films.

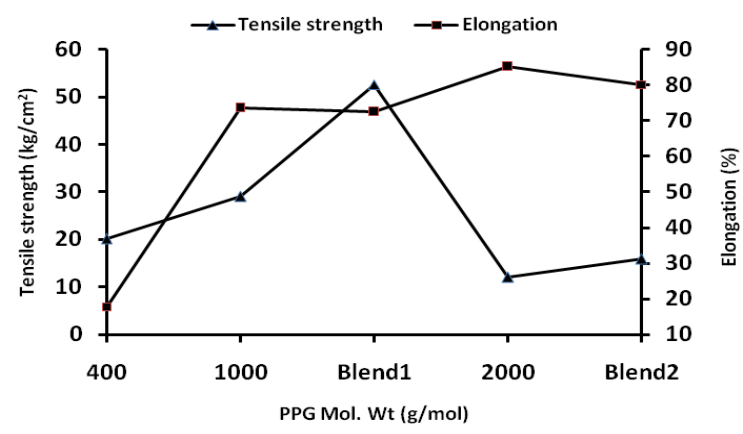

Blend 1 : PPG(1000:400) in 50/50 ratio, Blend2: PPG(2000:400) in 50/50

Fig-7: Effect of polyol molecular weight on tensile strength and percent elongation of the polyurethane film

\subsection{Influence of Soft Segment Length on the Thermal Properties}

The thermal properties of the synthesized prepolymer were investigated by DSC technique. For all the prepolymers only one $\mathrm{T}_{\mathrm{g}}$ was observed in the range from $-34.14^{\circ} \mathrm{C}$ to $-58.43^{\circ} \mathrm{C}$ and it was found to be decreasing with increasing molecular 
weight of the polyol (Fig- 8). This is because of the fact that as the soft segment length increases at a fixed hard segment length, there rise an increased tendency of the hard segment to be isolated in the domain of soft segment matrix. This phenomenon resulted in a higher degree of phase separation between hard and soft blocks, which produced a lower $\mathrm{T}_{\mathrm{g}}$ values. In the literature, it was reported that the phase separation was enhance by increasing soft segment length [6, $8,9]$.

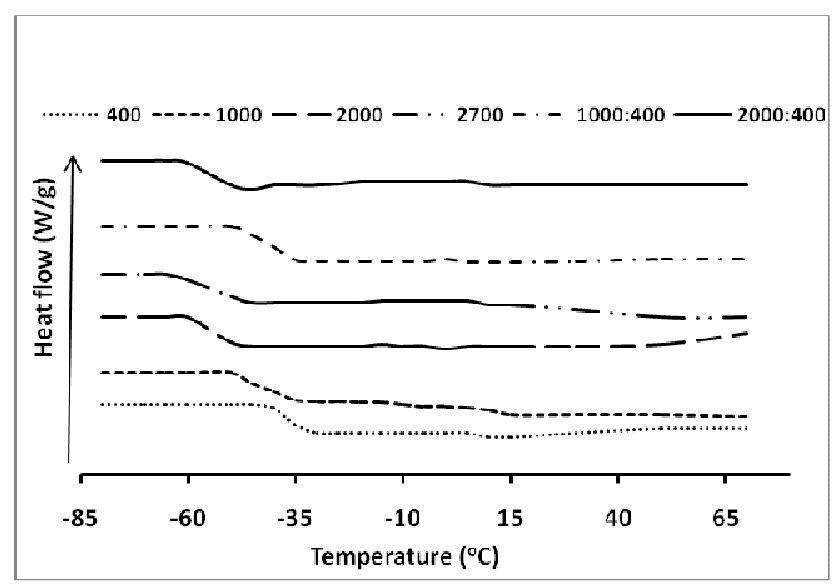

Fig-8 : DSC thermograms of the synthesized prepolymer

\section{CONCLUSIONS}

The isocyanate terminated polyurethane prepolymer was synthesized by thermal polymerization technique using MDI and PPG. Their formation was confirmed by FTIR which shows characteristics absorption bands of polyurethane. The Brookfield viscosity of the prepolymer decreases with increase in chain length of the soft segment, whereas the molecular weight and storage time was found to increases The curing time of the prepolymer was found to increase with increasing chain length of the soft segment i.e. with decrease in isocyanate content. The results on mechanical property shows that the tensile strength decreases with increasing molecular weight of the soft segment whereas opposite trend was observed in case of percent elongation of the polyurethane film. PPG 400 was found to produce polyurethane films having lower tensile strength and elongation due to its brittle nature. Mixing of PPG 1000 with PPG 400 in 50 / 50 ratio gives highest tensile strength among all the polyurethane films. Lower $\mathrm{T}_{\mathrm{g}}$ was observed in prepolymer having longer soft segment due to its flexible nature.

\section{ACKNOWLEDGEMENTS}

The authors wish to thank and acknowledge Indian Council of Medical Research, Govt of India for providing the financial support for carrying out the study.

\section{REFERENCES}

[1] Sonal Desai, I.M Thakore, B.D Sarawade, Surekha Devi, Effect of polyol and diisocyanates on thermomechanical and morphological properties of polyurethanes, European Polymer Journal 2000; 36, 711-725.

[2] Xia Dong Chen, Nan. Qiao Zhou and Hai Zhang, Preparation and properties of cast polyurethane elastomers with molecularly uniform hard segments based on 2,4-toluene diisocyanate and 3,5 dimethyl thiotoluenediame, J. Biomedical Science and Engineering. , 2, -253 .

[3] Rajendran G.P, Mahadevan V, Srinivasan M, Synthesis and properties of polyester based polyurethane. Eur.Polym.J. 1982, 18: 953-956.

[4] J.H Saunders and K.C Frisch, Polyurethane: Chemistry and Technology II, Technology, Interscience publisher, US, 1964, pg. 8-12.

[5] J.H Saunders and K.C Frisch, Polyurethane: Chemistry and Technology II, Technology, Interscience publisher, US , 1964 pg. 480.

[6] D.K Chattopadhyay, K.V.S.N Raju, Structural engineering of polyurethane coatings for high performance applications, Prog. Polym. Sci. 2007, 32 : 352-418.

[7] Buckanin R.S, Catalyst for the curing of a water curable isocyanate functional prepolymer. U.S. Pat 4,705,840, 1987.

[8] Sarawut Rimdusit, Tharathon Mongkhonsi, Pakawan Kamonchaivanich, Kuljira Sujirote, Sunan Thiptipakornl, Effect of polyol molecular weight on the properties of benzoxazine urethane polymer alloys, Polymer Engineering and Science, Nov 2008, 48(11) : 2238-2246.

[9] Sebastian Clau, Dirk J. Dijkstra, Joseph Gabriel, Oliver Klausler, Mathias Malner, Walter Meckel, Peter Niemz, Influence of chemical structure of PUR prepolymer on thermal stability, International journal of adhesion and adhesives, 2011, 31 : 513-523. 